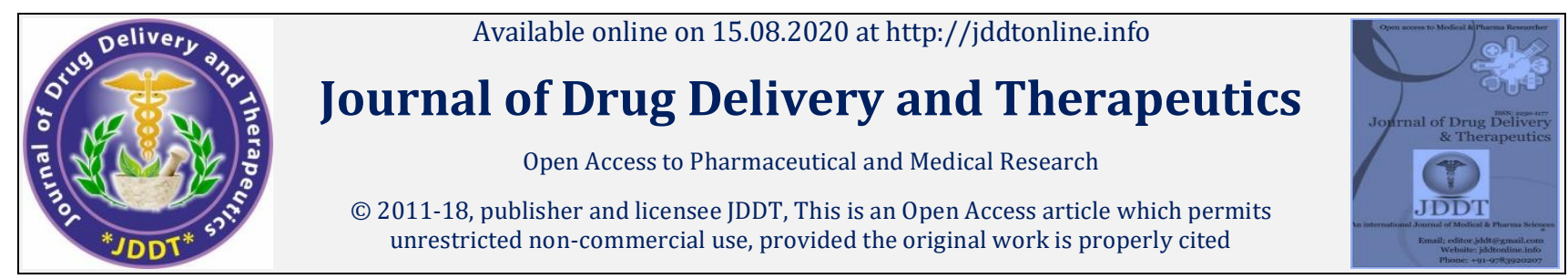

Open $\odot$ Access

Case Report

\title{
A Case Report on Iactrogenic Cushing Syndrome with Scabies and Oral Candidiasis
}

\author{
Md Abdullah Faizan*, Mohammed Baleeqhuddin, Salman Mir Ali
}

Department of Pharmacy Practice, Deccan School of Pharmacy Nampally, Hyderabad, Telangana 500001, India

\begin{abstract}
Cushing syndrome is a condition characterized by high blood levels of cortisol or other exogenous compounds of glucocorticoids. This can be iatrogenic or endogenous cortisol secretion, due to the either an adrenal tumor or hyper secretion of adrenotropic hormone by the pituitary gland. Due immunosuppression patient are prone to more infectious diseases. Here the patient is diagnoses with oral candidias is and scabies. Clinical investigation was carried out and laboratory investigations like Cortisol level in morning and evening, complete blood picture. He was provided with treatment of hydrocortisone, anti-fungal agents, anti-parasitic agents and skin moisturizer.
\end{abstract}

Keywords: Iactrogenic Cushing syndrome, Candidiasis, Scabies, Cortisol, Corticosteroid.

Article Info: Received 11 June 2020; Review Completed 14 July 2020; Accepted 22 July 2020; Available online 15 August 2020

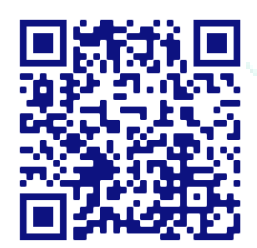

Cite this article as:

Faizan MA, Baleeqhuddin M, Ali SM, A Case Report on Iactrogenic Cushing Syndrome with Scabies and Oral Candidiasis, Journal of Drug Delivery and Therapeutics. 2020; 10(4-s):217-219 http://dx.doi.org/10.22270/jddt.v10i4-s.4271

*Address for Correspondence:

Md Abdullah Faizan, Department of Pharmacy Practice, Deccan School of Pharmacy Nampally, Hyderabad, Telangana 500001, India

\section{INTRODUCTION}

Cushing syndrome is a condition characterized by high blood levels of cortisol or other exogenous compounds of glucocorticoids. This can be iatrogenic or endogenous cortisol secretion, due to either an adrenal tumor or hypersecretion of adrenotropic hormone by the pituitary gland. Prolonged administration of exogenous glucocorticoid hormones especially in the treatment of lymph proliferative disorders at levels of higher doses are the most common cause of Cushing syndrome. The most common systemic side effects of prolonged use of glucocorticoids includes Cushing's syndrome, cataract, hypertension, dyslipidemia, skin atrophy, failure to thrive, hypothalamic pituitary adrenal axis suppression, striae, glaucoma and a predisposition to life threatening infections. ${ }^{1}$

Adverse drug reactions have been accounted over $6 \%$ of the total hospital admissions, these results in increase economic burden on health care system which is turn results in withdrawal of drugs from market 2

Many patients who develop iatrogenic Cushing's syndrome do so after receiving high doses of steroid over long periods of time. Therefore, the clinical manifestations can be more striking than those of spontaneous Cushing's syndrome, which tend to occur more gradually. The traditional stigmata include weight gain, usually presenting as central obesity with redistribution of body fat to truncal areas and the appearance of dorsocervical and supraclavicular fat pads and the classic moon face. Plethora, easy bruising, thin skin, striae, myopathy, and muscle weakness (particularly proximal muscles) can be seen. Patients are susceptible to poor wound healing and increased incidence of infection 3 .

Corticosteroids taken by mouth can increase your chance of getting a serious fungal infection. Oral corticosteroids include the following: Budesonide, Cortisone, Dexamethasone, Hydrocortisone, Methylprednisolone, Prednisolone, Prednisone, Triamcinolone

\section{CASE REPORT}

A 60 year old female diabetic patient was brought to emergency department having chief complaints of swelling all over the body since 4 months, fever since 2 days associated with chills and rigor, joint pain since 3 years SOB since 2 months, 3 episodes of vomiting in 3 days, pedal edema, white patches and facial puffiness.

On examination moon like face, hump on the nape, papillary lesions on neck and abdomen associated with itching, multiple erythematous nodules, web spacing fingers

Past medication history: -

DM: metformin500mg, glimepiride 1mg, insulin 
HTN: telmisartan $40 \mathrm{mg}$,

Joint pain: prednisolone $20 \mathrm{mg}$

Laboratory investigation

Cortisol level (morning): -252 nmol/L (145.4-619.4 nmol/L)

Cortisol level (evening): -411.22 nmol/L (94.9-462.4 $\mathrm{nmol} / \mathrm{L}$ )

White blood cells- 12100 cells/cumm

Ultrasound imaging of abdomen: - impression of fatty infiltration of liver.

Liver function test were normal

Renal function test was normal

Differential diagnosis: -

Physical examination of exogenous Cushing syndrome like moon shape face, fat distribution, weight gain, thin skin, proximal myopathy, muscle weakness (proximal muscles).

Physical examination for scabies like rashes and intense itching worsen at night, pimple like bumps, web spacing in fingers, multiple erythematous lesions.

Physical examination for oral candidiasis like white patches on inner cheeks, tongue, roof of the mouth.

Cortisol levels are been examined for exogenous Cushing syndrome.

Treatment: -

Upon admission to emergency department patient was provided with intravenous etoperidone $1 \mathrm{gm}+$ sulbactam $500 \mathrm{mg}$ twice a day, intravenous ondansetron $4 \mathrm{mg}$ thrice a day, intravenous furosemide $20 \mathrm{mg}$ twice a day, intravenous fluconazole $150 \mathrm{mg}$ once a day, tablet paracetamol $500 \mathrm{mg}$ thrice a day, Candid mouth wash, tablet Ivermectin $12 \mathrm{mg}$ weekly once, tablet hydroxyzine $10 \mathrm{mg}$ at bed time, Intravenous hydrocortisone $50 \mathrm{mg}$ twice a day, Permethrin $5 \% \mathrm{w} / \mathrm{w}$ cream and soap and skin moisturizer. The patient was isolated as a precaution measure.

Outcome: The patient has been under observation for 5 days. The patient has improved symptomatically. The patient was provided with steroid therapy by tapering the dose and closely monitoring the patient.

\section{DISCUSSION}

Glucocorticoids are commonly used in clinical practice for the management of various autoimmune, inflammatory and allergic diseases. Irrational administration of glucocorticoids is most common particularly in chronic therapies which lead to many side effects like hypothalamic pituitary adrenal axis suppression, Cushing's syndrome, increased risk of infections and changes in 142 mental status. The pharmacokinetic properties of the glucocorticoids, individual difference in steroid metabolism, daily dosage and the duration of treatment are the factors influencing therapeutic and adverse effect of glucocorticoids. 4 Before initiating steroid therapy, patients should be well informed about the possible side effects of steroids. Otherwise it may lead to severe systemic side effects including Cushing's syndrome, hypertension, dyslipidemia, suppression of hypothalamic pituitary adrenal axis, striae, glaucoma, skin atrophy, cataract and predisposition to life threatening infections. 4

Treatment is done by tapering the dose of corticosteroids which may take a year. Sudden stoppage corticosteroids after chronic intake can results in adrenal crisis. Slowly tapering the corticosteroid that is causing Cushing syndrome can help reverse the effects of adrenal gland atrophy 5

Scabies is an infestation of the skin by the mite $S$. scabies. Transmission is by direct skin-to-skin contact or indirectly through fomites. Symptoms typically appear 3 to 6 weeks after an infestation. However, in patients with a previous exposure to the mite, symptoms can appear as early as 24 hours post exposure. Lesions consist of pruritic erythematous papules with excoriations. They're usually symmetrical and involve the interdigital webs, the flexural aspect of wrists, the axillae, the peri-umbilical area, the elbows, the buttock, the feet, the genital area in males, and the peri-areolar area in females. The whole body-including the face and the scalp-can be involved in infants, the elderly and immunocompromised individuals 6

Oral candidiasis is generally obtained secondary to immune suppression, whether a patient's oral cavity has decreased immune function or if it is systemic. An example of local immunosuppression is the use of inhaled corticosteroids (often in the preventive treatment of asthma and chronic obstructive pulmonary disease). This immunosuppression has been found to be dose-dependent. For this reason, patients using such medications are instructed to rinse their mouth with water after each use. In this way, the beneficial effects of the corticosteroid affect the bronchioles, but the negative immunosuppressive effects become decreased in the oral cavity.

In clinical experience, this has been especially problematic with prednisone. Nonetheless, in most cases of exogenous Cushing's syndrome, the morning serum cortisol is found to be remarkably low, especially given the setting of Cushingoid symptoms. Corticotropin (ACTH) levels also should be relatively low, as pituitary production will be suppressed by exogenous steroids. The suppression of ACTH leads to atrophy of the adrenal cortex, and thus stimulation with centrepin should result in a decreased or absent plasma cortisol response. In some cases, diagnosis of exogenous Cushing's syndrome has been aided or confirmed by measurement of the glucocorticoid in question, although this may require specialized laboratory analysis 7,8

Iatrogenic Cushing syndrome is usually caused by extended use of oral or intravenous steroids of a period of time leading to increased cortisol level, which is followed by symptoms. The patients with Iatrogenic Cushing syndrome should be cautiously treated with steroid therapy and tapering it over a period of time. Patient are immunosuppressed and more vulnerable to infections.

The patient here is infected with oral candidiasis and scabies which are confirmed by physical examination. As the patient is more vulnerable, she must be extra cautious. The patient must maintain hygiene by washing body, by using mouth wash.

\section{ACKNOWLEDGEMENT}

Authors would like to thank the staff of the Department of general medicine, Owaisi hospital and research centre.

\section{REFERENCES}

1. Siddarama R, Reddy YH, Reddy GA. A case report on steroid induced Cushing syndrome and NSAID induced bronchial asthma. IAJPR. 2015; 5(4):1404-07.

2. Sateesh Kumar Reddy K, Ushasree P, Shanmuga Kumar SD, Goud SA. Prednisolone Induced Iatrogenic Cushing's Syndrome Associated with Secondary Diabetes: A Case Report. J Basic Clin Pharma 2018; 9:111-112.

3. Lionakis MS, Kontoyiannis DP. Glucocorticoids and invasive fungal infections. Lancet 2003;362(9398):1828-38 
4. Romanholi DJ, Salqadol LR. Arq Bras Endocrinol Metabol. 2007; 51(8):1280-92

5. Wisse B, Zieve D, Black B. Cushing syndrome - exogenous. US national library of medicine. American Accreditation HealthCare Commission.

6. Khalil S, Abbas O, Kibbi AG, Kurban M. Scabies in the age of increasing drug resistance. PLoS Negl Trop Dis. 2017; 11(11): e0005920. Published 2017 Nov 30.
7. Villanueva RB, Brett E, Gabrilove JL. A cluster of cases of factitious Cushing's syndrome. Endocr Pract 2000; 6(2):1437.

8. Krapf R. Development of Cushing's syndrome after use of an herbal remedy. Lancet 2002; 360:1884 